\title{
Applying the IoT platform and green wave theory to control intelligent traffic lights system for urban areas in Vietnam
}

\author{
Cao Tho Phan', Duy Duong Pham², Hoang Vu Tran ${ }^{3}$, Trung Viet Tran and Phat Nguyen Huu \\ ${ }^{1,2,3}$ The University of Danang - University of Technology and Education, Vietnam \\ 1[e-mail: pctho@ute.udn.vn], [e-mail: duyduongd2@gmail.com], [e-mail: thvu@ute.udn.vn] \\ ${ }^{4}$ The University of Danang - University of Science and Technology,Vietnam \\ ${ }^{4}$ [e-mail: trung-viet.tran@hotmail.com] \\ ${ }^{5}$ Hanoi University of Science and Technology, Vietnam \\ [e-mail: phat.nguyenhuu@hust.edu.vn] \\ *Corresponding author: Hoang Vu Tran
}

Received April 18, 2018; revised June 6, 2018; revised July 9, 2018; accepted September 10, 2018; published January 31, 2019

\begin{abstract}
This paper proposes an intelligent system performing an application with assistance of an Internet of Things (IoT) platform to control a traffic lights system. In our proposed systems, the traffic lights can be remotely controlled through the Internet. Based on IoT platform, the traffic conditions at different intersections of roads are collected and the traffic lights are controlled in a central manner. For the software part, the algorithm is designed based on the green wave theory to maximize the green bandwidth of arterial roads while addressing a challenging issue: the rapid changes of parameters including cycle time, splits, offset, non-fixed vehicles' velocities and traffic flow along arterial roads. The issue typically happens at some areas where the transportation system is not well organized like in Vietnam. For the hardware part, PLC S7-1200 are placed at the intersections for two purposes: to control traffic lights and to collect the parameters and transmit to a host machine at the operation center. For the communication part, the TCP/IP protocol can be done using a Profinet port embedded in the PLC. Some graphical user interface captures are also presented to illustrate the operation of our proposed system.
\end{abstract}

Keywords: IoT, ITS, intelligent traffic light, green wave, Internet of Things

This research is carried out in the framework of the project funded by the Ministry of Education and Training (MOET), Vietnam with the title "Research on the application of intelligent traffic systems in traffic management in some major urban cities in The Centre of Vietnam" under the grant number CNTT-02. The authors would like to thank the MOET for their financial support 


\section{Introduction}

Internet of Things (IoT), which can be regarded as an enhanced version of machine-to-machine communications, was proposed to realize intelligent thing-to-thing communications by utilizing the Internet infrastructure. The IoT is a totally interconnected world of devices with different shapes, sizes and manufactures. In addition, their "smart" capabilities allow them to communicate and interact with each other to exchange data. Each of them can make autonomous decisions and perform useful tasks based on a predefined set of conditions. The technologies operating on an IoT platform will bring many benefits in terms of making human life more comfortable, safer, easier and even richer. The IoT has begun to shape our modern world, where smart devices interact not only with humans, but also with many other smart devices attached to different objects, at different environments, and in different infrastructure [1]. How to effectively control a traffic lights system with assistance of IoT technologies become a popular research topic [2-4]. In this paper, based on the green wave theory and under an IoT platform, we propose to implement a system applying for Intelligent Traffic System (ITS) in urban areas in Vietnam.

The issue of traffic congestions at intersections has been known in many large cities around the world. The consequence would be causing accidents and wasting human's time. Accordingly, finding solutions to the issue has attracted the attention of many researchers. A method of intelligent signal control can offer an effective and efficient solution to some certain extend. Popular signal control methods can be classified as two categories: the isolated intersection and the coordinated control. The isolated intersection control is applicable if few interactions exist between two consecutive intersections. The strategy includes fixed-time control [5], which resorts to the statistics of history traffic-flow pattern, traffic-responsive control [6], which resorts to a real time traffic data to optimize the green time of a traffic light, and some other recent signal control strategies as proposed in [7-8]. Coordinated control strategies can be divided further into two sub-categories: fixed-time coordinated control and coordinated traffic-responsive strategies. Typical examples of the former strategy include bandwidth oriented signal timing MAXBAND [9], MULTIBAND [10] and network traffic control system TRANSYT. On the other hand, leading examples of the latter strategy include SCOOT and SCATS. In addition, we would mention a class of model-based traffic-responsive control strategies that emerged in the 1990s like OPCA, PRODYN, or RHODES. However, a large city with many heavy-traffic nodes, the applicability of such conventional strategies is rather limited due to computational complexity. Among coordinated control strategies, wave coordination control recently attracts much attention. Observing the predominance of traffic flow along arterials, the strategy then can provide numerous advantages [10]: (1) permission for a higher overall speed and a less number of stops, (2) fewer accidents due to lower red signal violations and rear-end collision probabilities since platoons of vehicles arrive at each green signal, (3) greater obedience from motorists and pedestrians to signal commands vehicles are more tightly spaced; hence motorists try to stay within green intervals while pedestrians stay at the curb. Green wave theory to control a real traffic system in developed countries could be easily applicable due to the availability of several stable parameters such as: intersections' distance, vehicles' velocity and traffic flow along arterial roads. However, those parameters would become rapidly varying in some areas including the urban areas in Vietnam. Hence, a real-time solution implemented for a green wave based method in controlling the traffic in Vietnam is highly demanded. 
This paper proposes a novel approach for an urban traffic network control in Vietnam by resorting to the green wave coordination technology. The system is implemented based on an IoT platform to remotely monitor and control traffic lights. Unlike the solutions proposed in [9-12] which were only designed for an individual and isolated intersection, our solution is capable in remotely controlling and monitoring a network of multiple intersections. In our system, inspired by the research results [13-15], algorithms are also designed under various scenarios. For examples, in [12] a method for urban arterial roads was proposed, in [11] a case that included an emergency vehicle and the tracking of a stolen vehicle based on RFID was handled, while [15] exploited an internet of vehicles to solve traffic control in multiple intersections. Note that the algorithm embedded in our hardware is very suitable for real traffic situations like in Vietnam.

The remainder of this paper is organized as follows. An overview of the proposed system is presented in Section 2, following by a communication procedure for remote monitoring and controlling in Section 3. In Section 4, we present our proposal of a green wave based approach applicable to Vietnam situations. For a further detail, the accompanied software is then presented in Section 5. Finally, Section 6 concludes the paper with some remarks.

\section{Overview System}

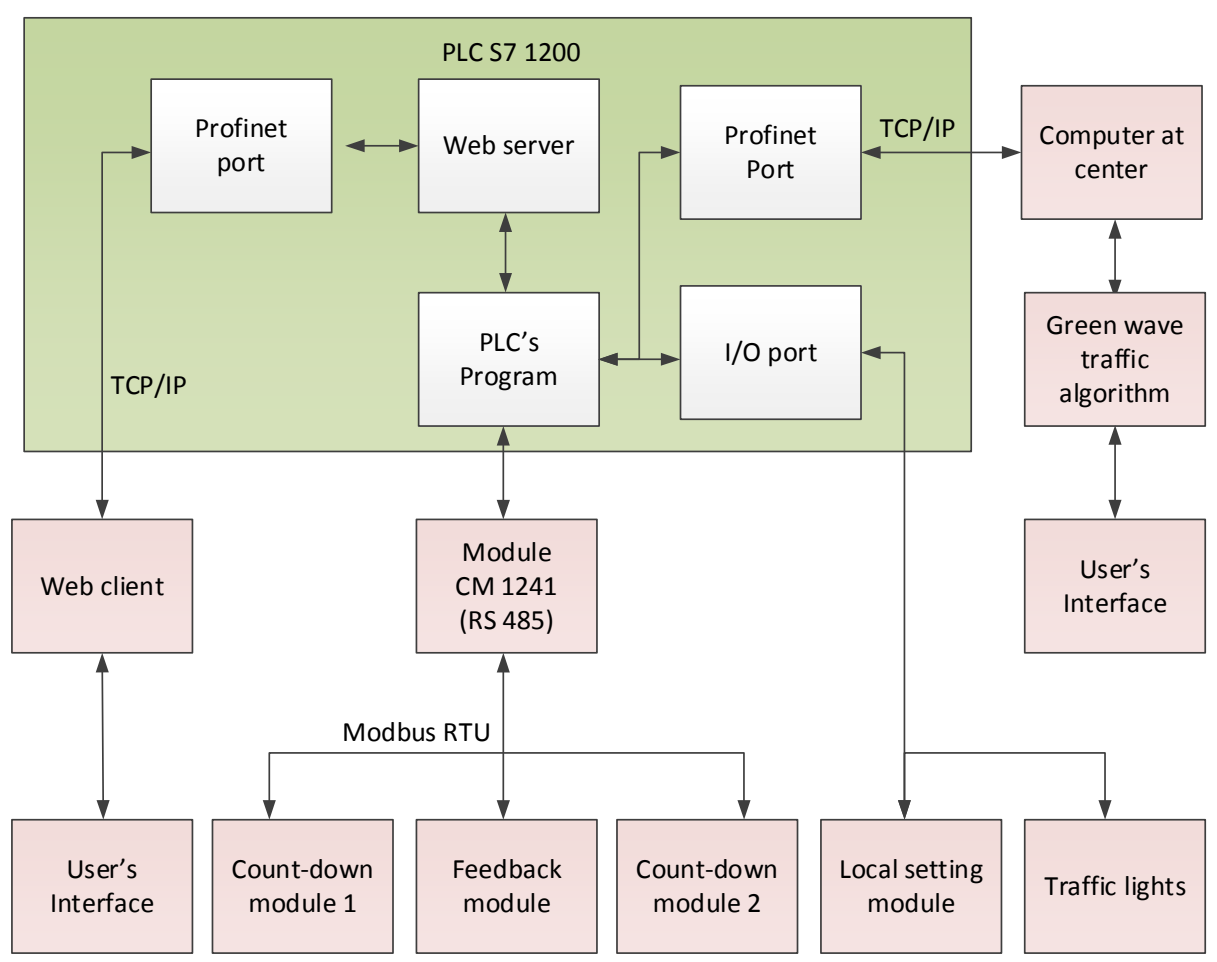

Fig. 1. Overview system via IoT platform

The overview of the proposed system is shown in Fig. 1, in which the detail of hardwave connection is presented. To build a IoT system, a PLC S7-1200 is used to control a traffic lights system at each intersection. The PLC embeds a Profinet port and a web-server module which are suit for Internet application. The communication between the PLC and a host computer at a control center (host machine) can be done using TCP/IP (Transmission Control Protocol/ Internet Protocol) protocol through a Profinet port of the PLC. Operation modes and 
parameters of every traffic-lights system are computed using algorithms in the host machine and tranfered to PLC at each intersection.



Fig. 2. Operation algorithm of the PLC

To transfer parameters between the PLC and function modules (feedback module, and count-down modules and local setting module), a RS-485 module (CM 1241) is added to the 
PLC. In which, the feed back module uses current sensors to feed back the status of traffic lights, count-down modules use 7-segments to show the remaining time of lights of each route and setting module is use to local setup parameters and working mode for traffic light system at each intersection. RS-485 supports inexpensive local networks and multidrop communications links, using the same differential signaling over twisted pair. It is generally accepted that RS-485 can be used with data rates up to $10 \mathrm{Mbit} / \mathrm{s}$ or, at lower speeds, distances up to $1,200 \mathrm{~m}(4,000 \mathrm{ft})$ [16]. Since the distance the feed back and setting modules are in the same cabinet with the PLC while the largest distance between the PLC and the count-down module about $100 \mathrm{~m}$, RS-485 communiation is used for this system. Each function module is controlled by a microcontroller (AT89S52) and communicates with the PLC using Modbus RTU (Remote Terminal Unit) protocol. Using communication to update and transfer parameters between PLC and function modules is necessary for remote controlling. The hardware design for feedback module, count-down modules and local setting module use an IC MAX-485 for Modbus RTU protocol. The operation algorithm of the PLC can be summarized in Fig. 2.

Users can remotely control and monitor the system by using a user's interface built in the host machine (see Fig. 7) or a user's interface on a website built in a web-server module (see Fig. 9).

\section{Communication for the System}

As can be seen in Fig. 1, TCP/IP protocol is used to transfer data between a PLC at each intersection and the host machine, and Modbus RTU protocol is used to transfer data between a PLC and its function modules.

\subsection{TCP/IP Protocol for the System}

Transferred data between the PLC and host machine is done using TCP/IP protocol. The detail of TCP/IP communication for the PLC can be seen in [17]. In which, a static IP (Internet Protocol) is fixed for the host machine. Transferred data that a PLC sends to the host machine using the frame is shown in Table 1.

Table 1. Transferred data frame to host machine

\begin{tabular}{|c|c|c|c|c|c|c|}
\hline PARAMETER & PLC ID & $\begin{array}{c}\text { LIGHT } \\
\text { TIME }\end{array}$ & $\begin{array}{c}\text { LIGHT } \\
\text { CYCLE }\end{array}$ & $\begin{array}{c}\text { LIGHT } \\
\text { STATUS }\end{array}$ & $\begin{array}{c}\text { LIGHT } \\
\text { ERROR }\end{array}$ & $\begin{array}{c}\text { LIGHT } \\
\text { CLOCK }\end{array}$ \\
\hline SIZE & 2 BYTE & 6 BYTE & 1 BYTE & 1 BYTE & 1 BYTE & 2 BYTE \\
\hline
\end{tabular}

In which:

- PLC ID: Identification number of each PLC respective to each intersection. By using this parameter in the frame, host machine knows where the intersection is.

- LIGHT TIME: The turning intervals of traffic lights consisting of green, red and yellow lights in two routes.

- LIGHT CYCLE: Light cycle of traffic light system.

- LIGHT STATUS: Status (on/off) of traffic lights. Status of each light is denoted by a bit in 2 status bytes.

- LIGHT ERROR: Shows error lights. Error state of each light is denoted by a bit in 2 error bytes.

- LIGHT CLOCK: The remaining time of lights of each route. 
PLC at each intersection sends parameters to host machine every half second. In order to do this, the IP of the host machine is added to the TCP/IP setting of the PLC. The host machine will set-up a connection with the PLC using the PLC's IP and received port.

Since a PLC's IP can be changed during work, the host machine cannot automatically find out the IP. Thus, the host machine always listens to the received data from PLC and sets up a connection to each PLC. Each intersection is represented by a PLC ID in received data (see Table 1). To do this, we designed a monitoring and controlling interface (see Fig. 7) in host machine using Matlab.

Transferred data frame form the host machine to each PLC is shown in Table 2.

Table 2. Transferred data frame to PLCs

\begin{tabular}{|c|c|c|c|c|}
\hline PARAMETER & $\begin{array}{c}\text { LIGHT } \\
\text { TIME }\end{array}$ & $\begin{array}{c}\text { LIGHT } \\
\text { TIME }\end{array}$ & $\begin{array}{c}\text { LIGHT } \\
\text { CYCLE }\end{array}$ & CONTROL \\
\hline SIZE & 6 BYTE & 6 BYTE & 1 BYTE & 1 BYTE \\
\hline
\end{tabular}

In which:

- LIGHT CYCLE and LIGHT TIME are parameters as in Table 1.

- CONTROL is a byte consisting of mode code and control code sent from the host machine to PLCs.

There is no PLC ID in the transferred data frame because the host machine has already taken a connection to PLC using PLC's IP. As can be seen in the frame, the data from the host machine is only sent if there is something that must be changed (parameters, mode and control).

\subsection{Modbus RTU for the System}

Since the distances from a PLC to function modules is not too much large, the Modbus RTU protocol using a RS-485 cable are used for this purpose. In which, A PLC S7- 1200 with a CM1241 module is used as the master device and function modules are used as slave devices. The detail of Modbus RTU communication can be seen in [17, 18]. In the system, we use read function (function code 03 - see Table 4) and write function (function code 06 - see Table 3) in Modbus RTU protocol. In which, the write function is used to send the remaining time of lights of each route to count-down modules and light's intervals to the setting module while the read function is used to read setting parameter from local setting module.

Table 3. Write function of Modbus RTU for master device

\begin{tabular}{|c|c|}
\hline Parameters & Size \\
\hline Slave address & 1 byte \\
\hline $\begin{array}{c}\text { Function code } \\
(06)\end{array}$ & 1 byte \\
\hline $\begin{array}{c}\text { Register address } \\
\text { to write data }\end{array}$ & 2 byte \\
\hline Writed data & 2 byte \\
\hline CRC code & 2 byte \\
\hline
\end{tabular}


In which:

- Slave address: The address of slave. In this case, slaves are function module and each function module is identified by an unsigned char number. Since the size of the slave address is 1 byte, a master device (PLC) can work with 256 different slavers. By using slave address, a PLC can read and write data to each function module.

- Function code: A number representing to a function of Modbus protocol. The function can be a read or write function with one or multi byte. For writing a 2-byte holding register to a function module, the code is 06.

- Register address to write data: The addresses of registers in function modules will be used to contain the writing data. The register address present to which parameter is sent to a function module.

- Writed data: The data of parameters will be written to function modules.

- CRC code: The code for error checking.

Table 4. Request frame (master) and answer frame (slaver) for read data function

\begin{tabular}{|c|c|c|c|}
\hline \multicolumn{2}{|c|}{ Request frame of mater } & \multicolumn{2}{c|}{ Answer frame of slave } \\
\hline Parameters & Size & Parameters & Size \\
\hline Slave address & 1 byte & Slave address & 1 byte \\
\hline Function code (03) & 1 byte & $\begin{array}{c}\text { Function code } \\
\text { (03) }\end{array}$ & 1 byte \\
\hline $\begin{array}{c}\text { Address of register } \\
\text { will be read }\end{array}$ & 2 byte & $\begin{array}{c}\text { Number of data } \\
\text { byte (2xN). }\end{array}$ & 1 byte \\
\hline $\begin{array}{c}\text { Number of register } \\
\text { will be read }\end{array}$ & 2 byte & $\begin{array}{c}\text { Data of requested } \\
\text { register }\end{array}$ & $\begin{array}{c}2 x N \\
\text { byte }\end{array}$ \\
\hline CRC code & 2 byte & CRC code & 2 byte \\
\hline
\end{tabular}

In which:

- Slave address: The address of each slave (function module) as in Table 3.

- Function code: A number represent to a function of Modbus protocol as in Table 3.

For reading 2xN-byte holding register from a function module, the code is 03 .

- Address of register will be read: The first address of registers containing parameters in function modules that the maters (the PLC) will read.

- Number of register will be read: Number of registers containing parameters in function modules that the maters (the PLC) will read.

- Number of data byte $(2 \mathrm{xN})$ : number byte of parameters that the funtion module (respective to slave address) will send to the PLC.

- Data of requested register: 2xN byte of parameter that the funtion module (respective to slave address) send to the PLC.

- CRC code: The code for error checking. 


\section{Green Wave for Urban Traffic in Vietnam}

\subsection{Modbus RTU for the System}

The green wave model for traffic lights systems along an arterial road can be inferred from a green wave model for two adjacent intersections [19-21]. Thus, in this subsection, authors present a green wave model for two adjacent intersections first. Then the green wave optimization for traffic lights systems along an arterial road is inferred in the next subsection.

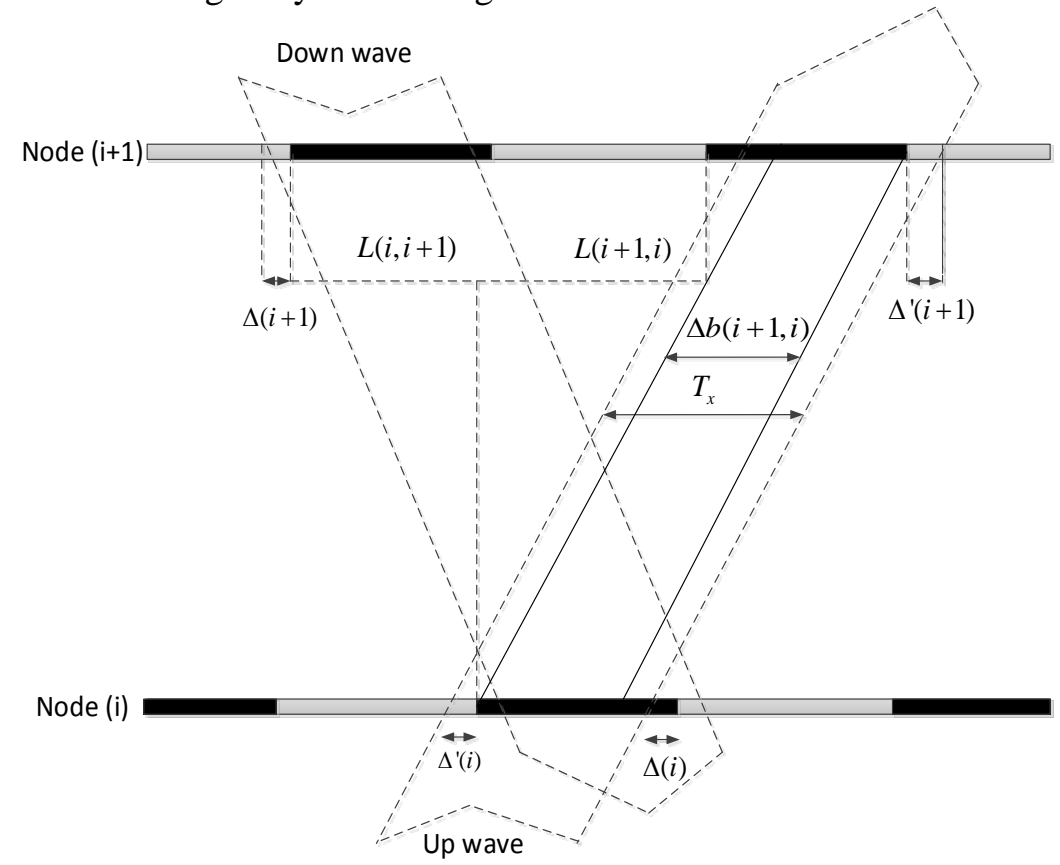

Fig. 3. Model for green wave

The green wave model for two adjacent intersections can be seen in Fig. 3. In which, the model shows the relationship between vehicle speed, traffic cycle, green light phase, intersection offset and traffic flow between intersection $i$ and intersection $i+1$. In the figure, green light interval $\left(T_{x}\right)$ is shown in black color while red light interval is shown in gray color at a node $i$.

Assume that a vehicle appears at intersection $i$ at $U(i), \Delta^{\prime}(i)$ is the waiting time for the green light to turn on, $T(i, i+1)$ is the running time from intersection $i$ to intersection $i+1$ and $\Delta^{\prime}(i+1)$ are the turn on time of the green light at intersection $i+1$ when the vehicle arrives there.

Similar to this for a vehicle which goes from intersection $i+1$ to intersection $i$, we have $U(i+1), \Delta(i+1), T(i+1, i)$ and $\Delta(i)$.

Let $L(i, i+1)$ and $L(i+1, i)$ be backward degrees of intersection $i$ in comparison with intersection $i+1$ and vice versa. In which, backward degrees [22] is used to accommodate green waves for travelling from intersection to intersection at a specied pace.

From Fig. 3, we have:

$$
\begin{aligned}
\Delta(i+1) & =-\Delta(i)+T(i, i+1)-L(i, i+1) \\
\Delta^{\prime}(i+1) & =-\Delta^{\prime}(i)+T(i+1, i)-L(i+1, i)
\end{aligned}
$$


Thus, the green bandwidth for "down-wave" between intersection $i$ and intersection $i+1$ is:

$$
\Delta b(i, i+1)=T_{x}-([\Delta(i+1)]+[\Delta(i)])
$$

Where $T_{x}$ is the turning on interval of the green lights and the green bandwidth $[9,23]$ is the green signal effect that a series of traffic lights are coordinated to allow continuous traffic flow over several intersections in one main direction.

Similarly, the green bandwidth for "up-wave" between intersection i and intersection $i+1$ is:

$$
\Delta b(i+1, i)=T_{x}-\left(\left|\Delta^{\prime}(i+1)\right|+\left|\Delta^{\prime}(i)\right|\right)
$$

To get the maximum green bandwidth, the intervals $([\Delta(i+1)]+[\Delta(i)])$ in (2) and $\left(\left|\Delta^{\prime}(i+1)\right|+\left|\Delta^{\prime}(i)\right|\right)$ in (3) must be minximum.

Let $D(i, i+1)$ is distance between intersection $i$ and $i+1$. Let $V(i, i+1)$ and $V(i+1, i)$ are the vehicle speed in direction from intersection $i$ to $i+1$ and vice versa.

We have

$$
\begin{aligned}
|\Delta(i+1)|+|\Delta(i)| & =|\Delta(i)|+|-\Delta(i)+D(i, i+1) / V(i+1, i)-L(i+1, i)| \\
\left|\Delta^{\prime}(i+1)\right|+\left|\Delta^{\prime}(i)\right| & =\left|\Delta^{\prime}(i)\right|+\left|-\Delta^{\prime}(i)+D(i, i+1) / V(i, i+1)-L(i, i+1)\right|
\end{aligned}
$$

From Fig. 3, we have

$$
L(i, i+1)+L(i+1, i)=C
$$

Where $C$ is the cycle time of the traffic light system ( $C$ is an assume value for green wave optimatization algorithm and increases from $C_{\min }$ to $C_{0}$ ).

In which, $C_{0}$ and $C_{\min }$ are maximum and minimum of signal cycle of green wave and computed as:

$$
\begin{gathered}
C_{\min }=\min \left\{C_{\min }^{i}\right\} \\
C_{\max }=\max \left\{C_{\max }^{i}\right\}
\end{gathered} \quad i=1: N
$$

$N$ is is number of intersection in green wave, $C_{\min }^{i}$ and $C_{\max }^{i}$ is function of trafic flow weight of intersection ith and calculate by Websters formula:

$$
C=\frac{1.5 G+5}{1-\sum_{j=1}^{t} q_{j} / S_{j}}
$$

where G is lost time of intersection, $q_{j}$ is the nominal inflow to link $j$ in vehicles per hour, $S_{j}$ is the saturation flow of link $j$ and $t$ is the set of all links which belong to intersection. The description more details in [24].

From (2), (3), (4) and (5) we can see that the green bandwidth is a function of cycle time $C$, vehicle speeds $(V(i, i+1), V(i+1, i))$ and backward degree between two intersections $(L(i, i+1), L(i+1, i))$.

\subsection{Green Wave Optimization}

In this subsection, we have to find out the value of cycle time $C$, vehicle speed $(V(i, i+$ 1), $V(i+1, i))$ and backward degree $(L(i, i+1), L(i+1, i))$ so that the right side of equation (4) is minimum. Green wave optimization must be suitable with the situation in urban areas. Thus, the condition in (6) must be satisfied:

$$
\begin{gathered}
|\Delta(i+1)| \leq 0.2 T_{x} \\
\left|\Delta^{\prime}(i+1)\right| \leq 0.2 T_{x} \\
|\Delta(i)| \leq 0.2 T_{x} \\
\left|\Delta^{\prime}(i)\right| \leq 0.2 T_{x} \\
\min \left(|\Delta(i+1) Q(i, i+1)|+\left|\Delta^{\prime}(i+1) Q(i, i+1)\right|\right) \\
\min \left(|\Delta(i) Q(i+1, i)|+\left|\Delta^{\prime}(i) Q(i+1, i)\right|\right)
\end{gathered}
$$


In which the fifth and sixth conditions in (6) are to minimize waiting time at nodes, $Q(i, i+1), Q(i+1, i)$ are respectively throughput of vehicles from the node $i^{\text {th }}$ to the $(i+1)^{t h}$ and vice versa.

To solve (6), we employ a heuristic approach. Variables like light period, speed of backward fluid between two nodes are gradually changed to find the maximum value of green bandwidth $(\max (\Delta b(i, i+1)+\Delta b(i+1, i)$ which ensures (6) and (7).

$$
\begin{aligned}
& \Delta b(i, i+1) \geq 0.6 T_{x} \\
& \Delta b(i+1, i) \geq 0.6 T_{x}
\end{aligned}
$$

After the green bandwidth between the nodes $i^{\text {th }}$ and (i+1)-th is computed, the same procedure is implemented for the nodes $i^{\text {th }}$ and $(i+1)^{t h}$ until the last nodes.

The procedure is described as the following:

- Step 1: Assume a light period $C\left(C_{\min }<C<C_{0}\right)$.

- Step 2: Assume a speed of vehicle fluid $(V(i, i+1), V(i+1, i))$, and a backward degree $(L(i, i+1), L(i+1, i))$ for/at the node i-th

- Step 3: Assume $|\Delta(i)| \leq 0.2 T_{x},\left|\Delta^{\prime}(i)\right| \leq 0.2 T_{x}$

- Step 4: Compute $\Delta(i+1), \Delta^{\prime}(i+1)$ using (4)

- Step 5: Compute time indexes in (6).

- Step 6: Change backward degree $(L(i, i+1), L(i+1, i)) ; \Delta(i)$ and $\Delta^{\prime}(i)$ and choose minimum values of $\Delta(i+1)$ and $\Delta^{\prime}(i+1)$

- Step 7: After choosing optimal backward degree and green bandwidth for a couple of nodes $\mathrm{i}, \mathrm{i}+1$, values of $\Delta(i+1)$ và $\Delta^{\prime}(i+1)$, are used in other couples of nodes, following step 2 to step 6 until the last node.

- Step 8: Speed of vehicle fluid $(V(i, i+1), V(i+1, i))$ is changed, then alter the period $C$ and repeat the previous steps until light period, speed of vehicle fluid, and backward degree are chosen to maximize the green bandwidth.

\subsection{Apply the Green Wave Theory for a Road}

We test the combining green wave algorithm for a route including 8 cross-overs on a main road in three different cases where distances between the nodes are altered as in the Table 5. Results of the algorithm in case 1, 2 and 3 are shown in the Fig. 4, Fig. 5 and Fig. 6, respectively.

Many problems existed are indicated the ratio between green wave bandwidth and cycle time vary from 0.1 to 0.4 [19-21] or the ratio between green wave bandwidth and green signal vary from 0.2 to 0.6 [19-21]. The best value for this ratio is 1 . In Vietnam traffic conditions, we propose value of this ratio greater than 0.6 for our procedure as in (7). To evaluate the performance of our green wave optimization algorithm, we use the ratio defined by

$$
\partial=\frac{T_{x}-\max (\Delta b(i, i+1)+\Delta b(i+1, i))}{T_{x}}
$$

As in conditions in (7), the green wave optimization is okay if $\partial \geq 0.6$. However, as can be seen in the table, the minimum value of the ratio is 0.93 . This is a very good value for green wave optimization. 
Table 5. Distribution of intersections along the priority route

\begin{tabular}{|c|c|c|c|c|c|c|c|c|}
\hline $\begin{array}{c}\text { Distance (m) } \\
\text { D(i,i+1) }\end{array}$ & $\begin{array}{c}\text { Node } \\
\mathbf{( 1 , 2 )}\end{array}$ & $\begin{array}{c}\text { Node } \\
(\mathbf{2 , 3})\end{array}$ & $\begin{array}{c}\text { Node } \\
\mathbf{( 3 , 4 )}\end{array}$ & $\begin{array}{c}\text { Node } \\
\mathbf{( 4 , 5 )}\end{array}$ & $\begin{array}{c}\text { Node } \\
\mathbf{( 5 , 6 )}\end{array}$ & $\begin{array}{c}\text { Node } \\
(\mathbf{6 , 7})\end{array}$ & $\begin{array}{c}\text { Node } \\
\mathbf{( 7 , 8 )}\end{array}$ & $\boldsymbol{\partial}$ \\
\hline Case 1 & 256.5 & 176 & 194.4 & 210.6 & 189 & 202.5 & 265.5 & 0.95 \\
\hline Case 2 & 156.5 & 200 & 194.4 & 210.6 & 160 & 220.5 & 206.5 & 0.93 \\
\hline Case 3 & 200 & 250 & 190 & 175 & 200 & 210.5 & 150.5 & 0.94 \\
\hline
\end{tabular}

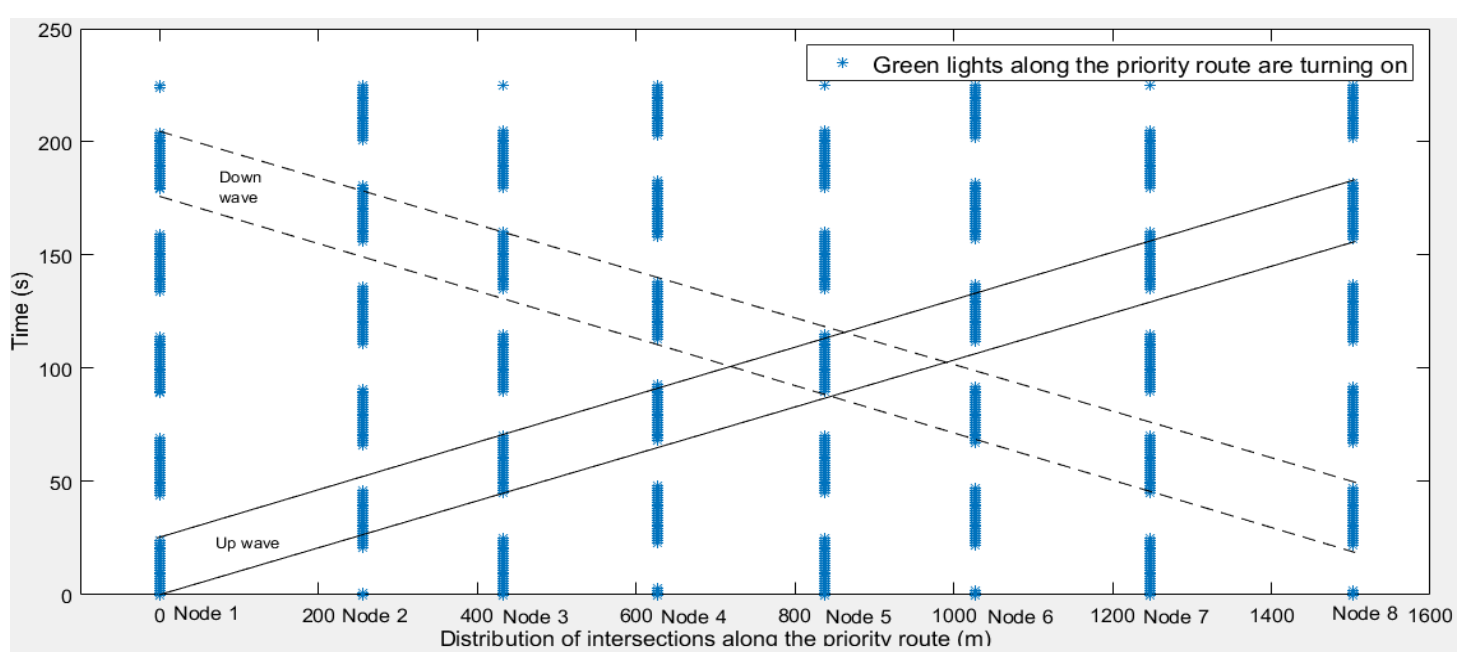

Fig. 4. Green wave combination for the first case (cycle time: $45 \mathrm{~s}$, vehicle speed: $41 \mathrm{~km} / \mathrm{h}, \partial=0.95$ )

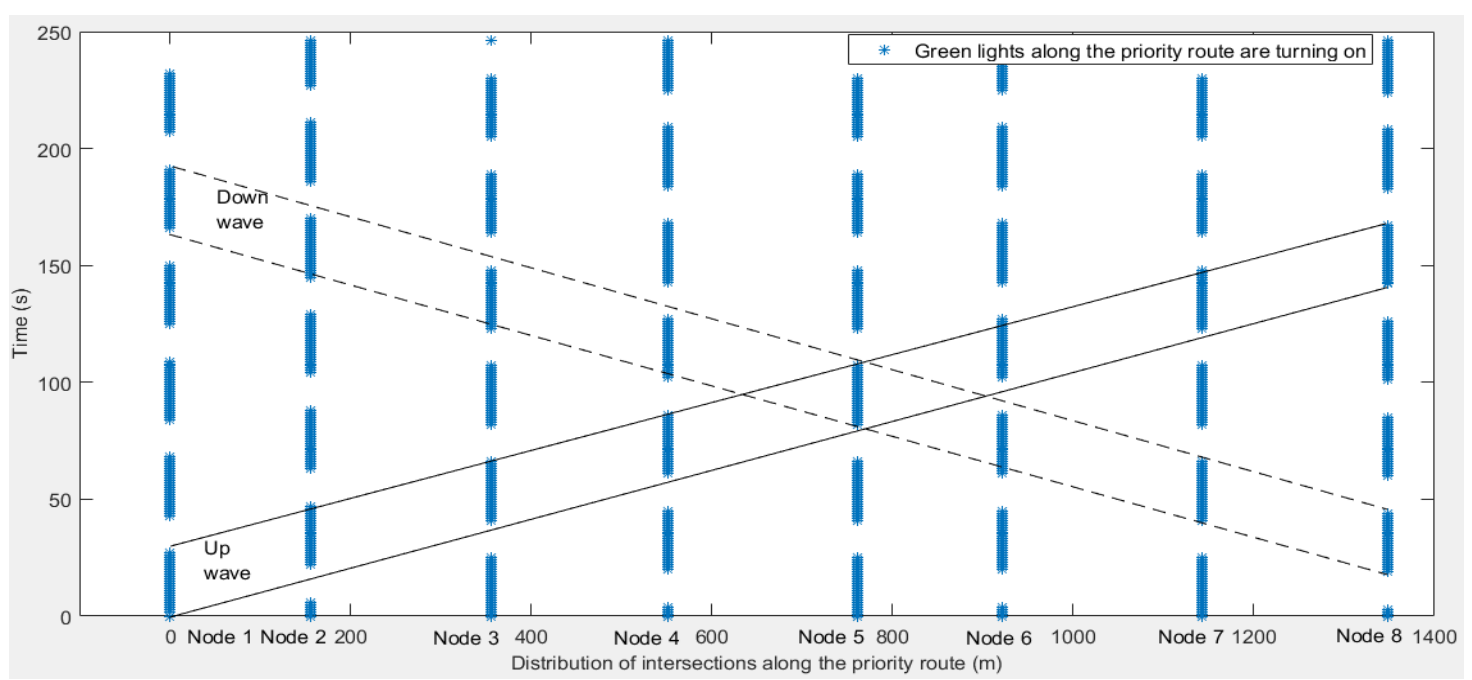

Fig. 5. Green wave combination for the second case (cycle time: $41 \mathrm{~s}$, vehicle speed: $32 \mathrm{~km} / \mathrm{h}, \partial=0.93$ ) 


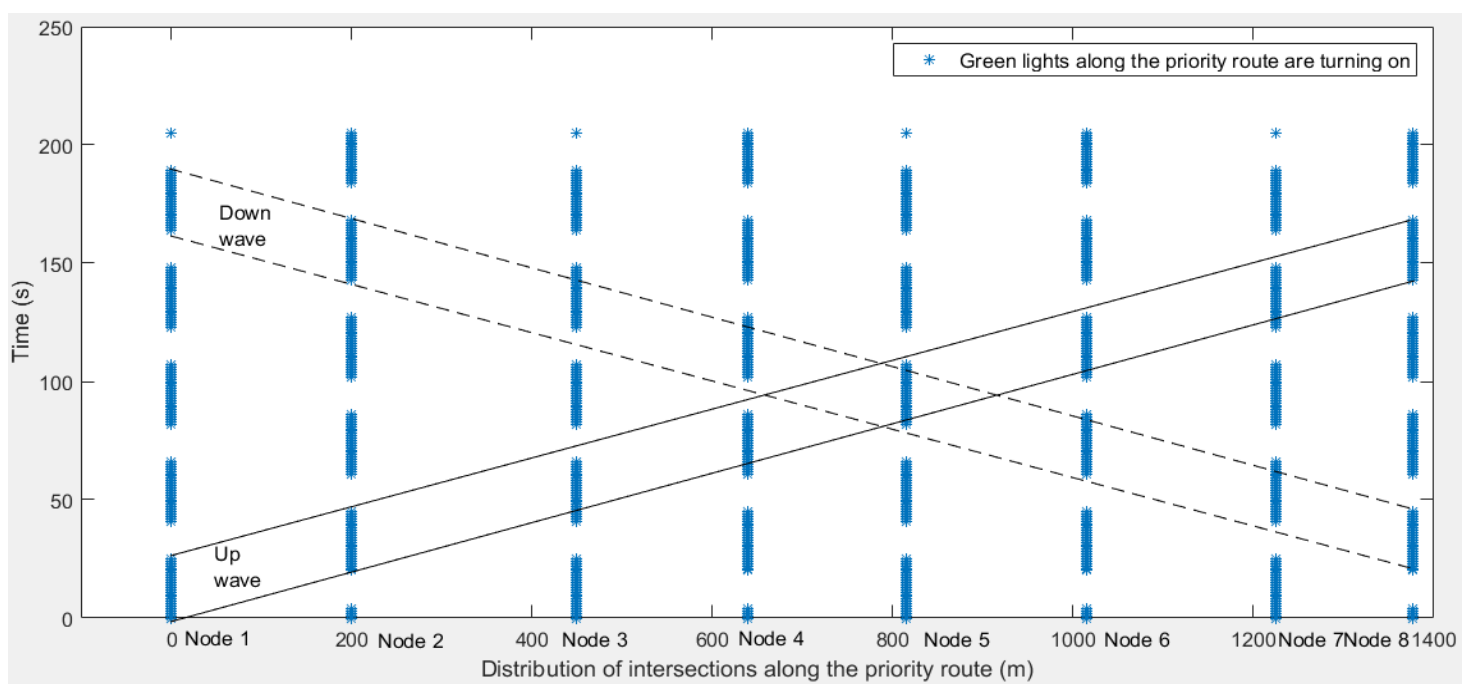

Fig. 6. Green wave combination for the second case (cycle time: $41 \mathrm{~s}$, vehicle speed: $37 \mathrm{~km} / \mathrm{h}, \partial=0.94$ )

\section{Software for Controlling and Monitoring Traffic Lights System}

The softwave is built for a host machine at a traffic light operation center using Matlab. In principle, using TCP/IP protocol and port connection after the host machine listens to received data (includes PLC's ID) (see Section 3), the host machine can connect to so many PLCs at intersections. However, in this paper, we implemented a traffic system with 07 PLC S7-1200 (present to 07 traffic lights system at intersection). Fig. 7 shows the monitoring and controlling interface in three cases as in Table 6. In which, the interface is separated into 10 numbered zones as can be seen in Fig. 7a.

Table 6. Distribution of intersections along the priority route for software test

\begin{tabular}{|c|c|c|c|c|c|c|c|}
\hline $\begin{array}{c}\text { Distance (m) } \\
\text { D(i,i+1) }\end{array}$ & $\begin{array}{c}\text { Node } \\
(\mathbf{1 , 2})\end{array}$ & $\begin{array}{c}\text { Node } \\
(\mathbf{2 , 3})\end{array}$ & $\begin{array}{c}\text { Node } \\
\mathbf{( 3 , 4 )}\end{array}$ & $\begin{array}{c}\text { Node } \\
\mathbf{( 4 , 5 )}\end{array}$ & $\begin{array}{c}\text { Node } \\
\mathbf{( 5 , 6 )}\end{array}$ & $\begin{array}{c}\text { Node } \\
\mathbf{( 6 , 7 )}\end{array}$ & Figure \\
\hline Case 1 & 200 & 180 & 250 & 200 & 250 & 180 & Fig. 10a \\
\hline Case 2 & 200 & 250 & 180 & 200 & 250 & 250 & Fig. 10b \\
\hline Case 3 & 180 & 250 & 300 & 200 & 250 & 200 & Fig. 10c \\
\hline
\end{tabular}

The CONNECT and DISCONNECT buttons in Zone 1 are used to start and stop the connection between the host machine and a PLC. After pressing the CONNECT button, the host machine begins listening to send data from PLC and sets up a connection with the PLC. Then "Connected" label is shown in the interface to notify that the setting process is finished. The DISCONNECT button is used to disconnect the connection between host machine and PLCs.

After that, we can remotely control and monitor the traffic lights system as an intersection one by one by choosing intersections in the pop-up menu (sec Zone 4). The information of the chosen intersection (such as IP, Port and intersection name, traffic lights system status) is shown below the pop up menu. The status of traffic lights and the count-down time for each router are shown in Zone 7.

An adjustment of working parameters of a traffic light system can be manually implemented by an installment in Section 2 where a traffic light at each node is independently or combined, according to green wave, monitored and controlled. Specifically, when an auto 
button is on, the software conducts the computation of the optimal period and phase contribution based on Webster formula, in which necessary parameters are defined in the Section 6 . When an auto button is off, working parameters are manually altered in the Section 3. The buttons START and STOP in the Section 5 will control an on/off status of a traffic light system at a node.

A guide user interface using the green wave (see Section 4) is presented in Section 8-10. Specifically, in Section 7, seven nodes are distributed along a main axis/road. Distances between nodes are proportional to that in reality and could be changed by inputs at Section 10 . At each node, an interface shows only a traffic light and its backward time.

After distance between nodes are plugged in in Zone 10, push the CALCULATE button to run the green wave algorithm in Section 4. Output of the algorithm, including a period of the traffic light system, assumed velocities of vehicles, and time differences between starting time indices at each node compared to the first node, are then shown by figures in Zone 9 and also in Zone 10.

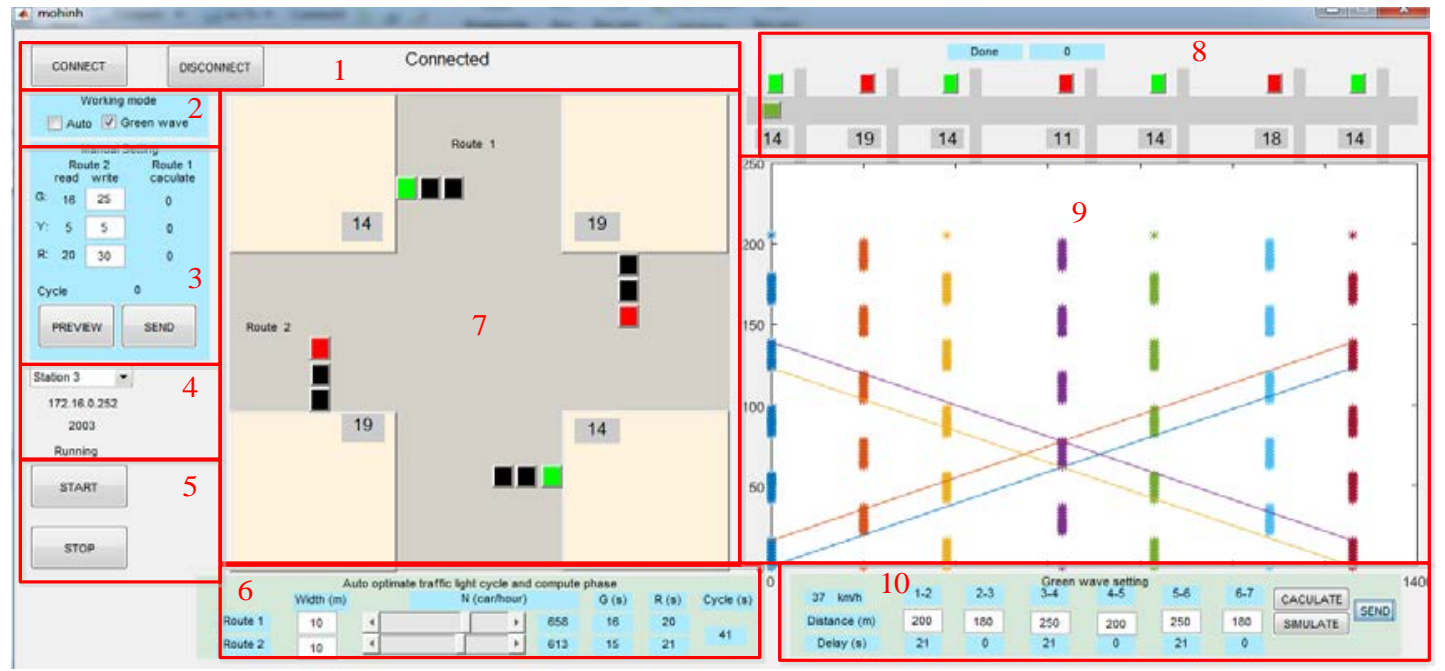

(a)

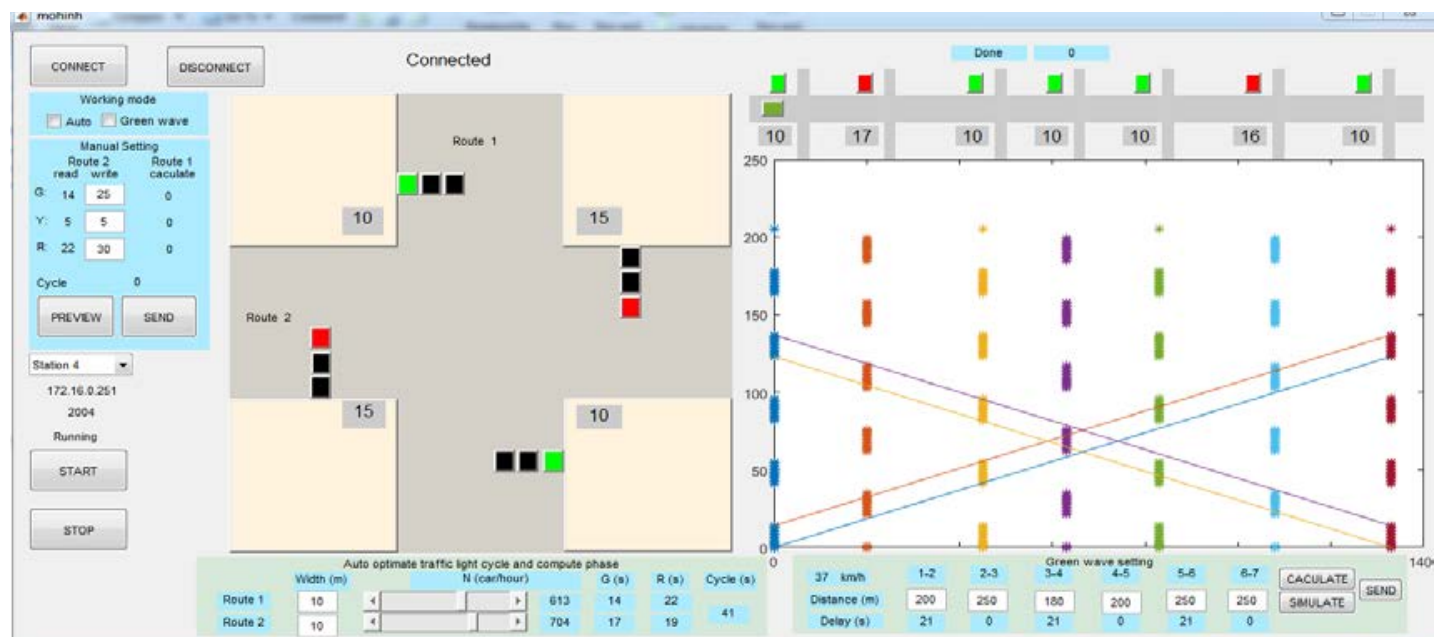

(b) 


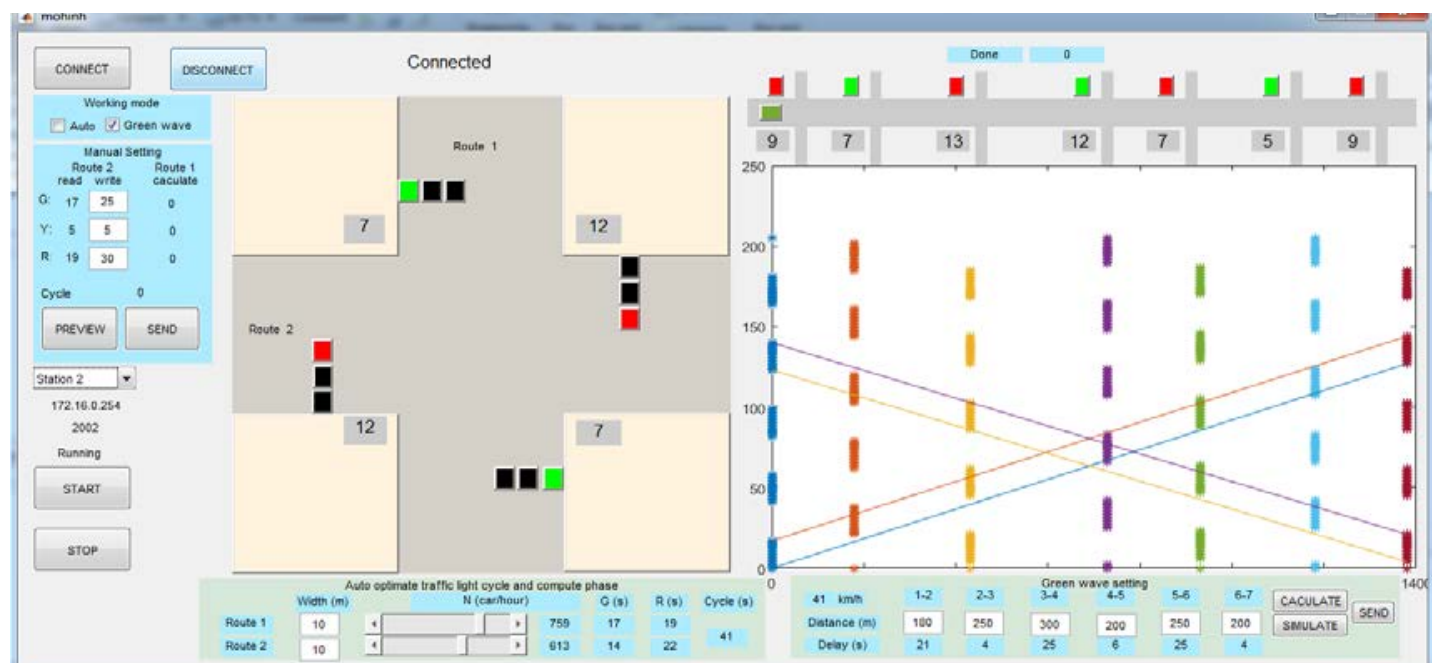

(c)

Fig. 7. Monitoring and controlling interface for intelligent traffic light system in Matlab

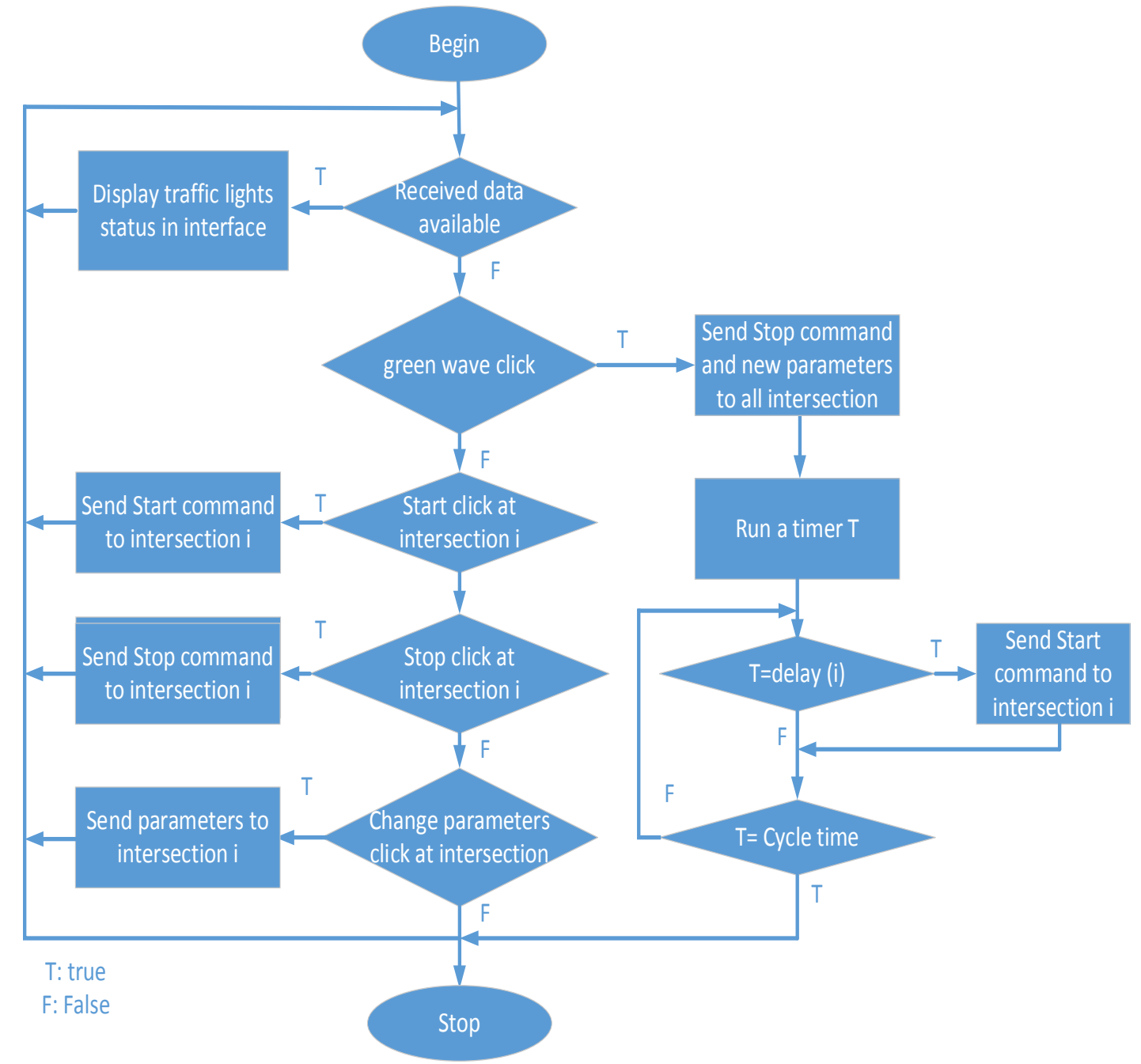

Fig. 8. Operation algorithm of monitoring and controlling interface 
After pushing the SEND button, the server will send a stop command to all traffic light systems at nodes along the main axis. The server then updates working parameters at each nodes including 'on' period, 'on' interval of the green light at each route. Finally, commands from the server sequentially activate traffic light system at each node. Time differences between commands are exactly the same as time differences between starting time indices at nodes compared to the first node (see section 10). During this process, the word "Processing" and the time delay, compared to the activated time instance at the first node, are represented in section 8. Status command changes to "Done" after the traffic light system at the last node is activated.

In addition to the above-mentioned functional buttons, a simulation of the system's operation is integrated and can be implemented by pushing SIMULATE button. For example, after pushing SIMULATE button, a vehicle, represented by a blue rectangle, will appear at the starting point and move to the end point with a designed speed. This vehicle will pass each node only when the traffic light at the node is green. Hence, the simulation reveals a real traffic situation and convinces users the validity of the system's working parameter as well as its operation.

As can be compared in Zone 8, 9 and 10 of figures, parameters (such as: distances, delay time and design vehicle flow) are changed depending on distances between intersections. In Zone 4 and 7, we show the traffic lights monitoring for intersection 3, 4 and 5 for Fig. 7a, Fig. 7b and Fig. 7c, respectively. The operation algorithm of the interface is summarized in Fig. 8.

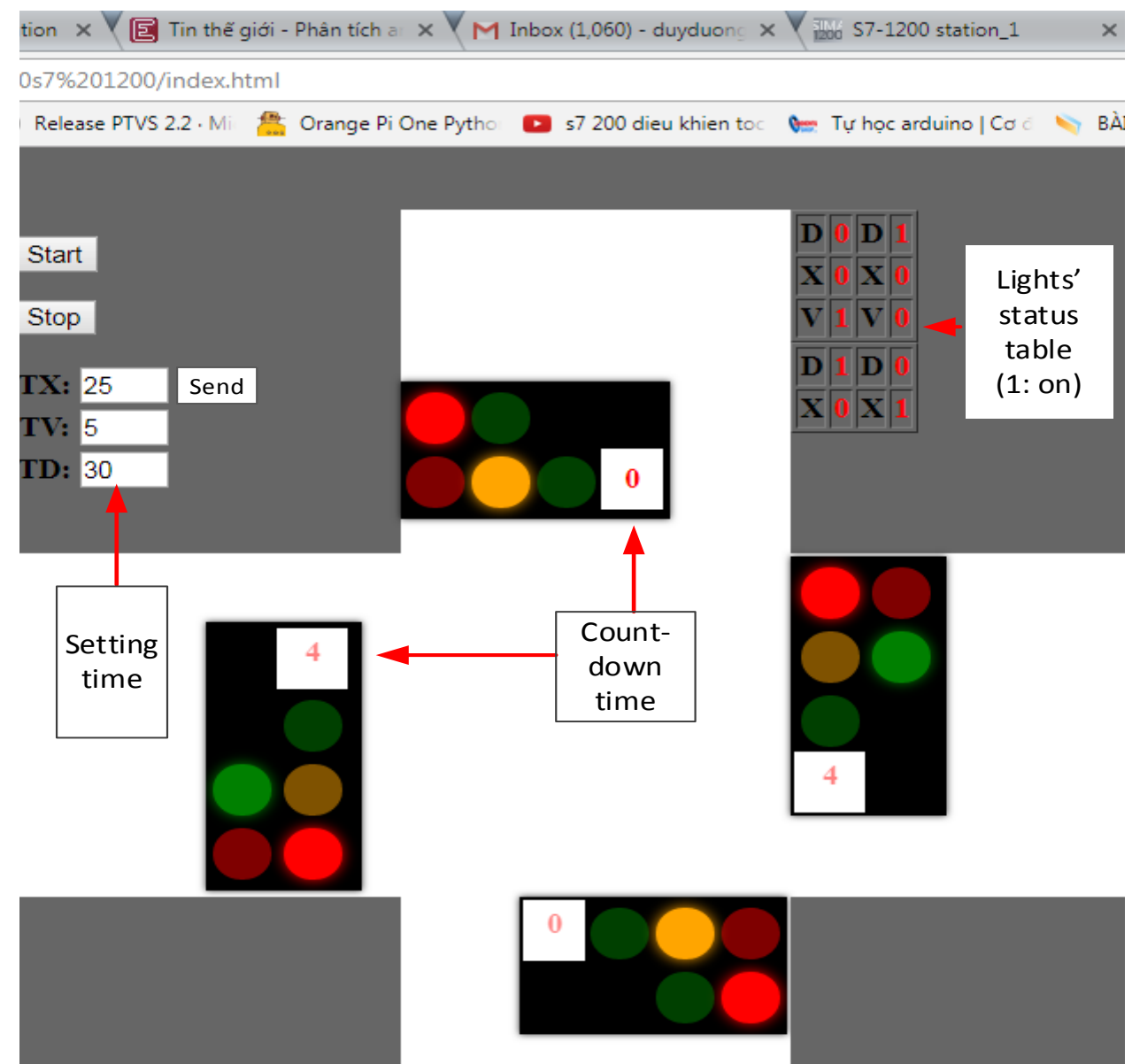

Fig. 9. Monitoring and controlling interface for intelligent traffic light system in website 
Fig. 9 shows the user's monitoring interface on web-site. By using the web-site, the user can remotely monitor the status of traffic lights, light's times and control traffic light system at each intersection. (such as: start/ stop the system, send setting times to the system). To access the interface, user have to login with user's name and password.

\section{Conclusion and Future Works}

This paper proposed an IoT application for controlling an intelligent traffic lights system. Basing on Internet connections between intersections, we applies the green wave theory along a arterial roads in a real traffic situation for urban areas in Vietnam; when problems arise in a real-time implementation of a green wave coordination control for traffic: changes in parameters like cycle time, splits, and offset and non-fixed vehicles' velocities and traffic flow along arterial roads should be considered.

The IoT application uses a PLC S7-1200 at every intersection to control traffic lights system, transfers parameters to the host machine and receives parameters from the host machine at the operation center using the TCP/IP protocol. The TCP/IP protocol can be done via a Profinet port embedded in the PLC. A communication module (CM 1241) is added to the PLC to communicate with function modules via a Modbus RTU protocol. Test-bed of a traffic light control cabinets using a PLC S7-1200 and function modules at a intersection are built to test working ability of the proposed hardware system.

Basing on the IoT platform we proposed a method to apply the green wave theory in urban areas of Vietnam so that the green bandwidth of arterial roads is maximied by changing cycle time, splits, and offset and non-fixed vehicles' velocities and traffic flow along arterial roads.

Futhermore, an interface for monitoring and intelligent controll the traffic light system is built in Matlab. From the interface, we can see status of traffic lights, cycle time and light interval at each isolated intersection, and control all traffic lights system connected with the host machine. By using the interface, user can apply a green wave theory for intersections along an arterial road for urban areas in Vietnam.

In this paper, authors combine the IoT platform and green wave theory for a single arterial road. In the future work, authors will propose a method to apply the system for a network of arterial roads which is popular in big cities. Futhermore, the real test in real environment in some complex urban areas should be done to evaluate the stability and effectiveness of the system. Thus, the result of this paper will be improved and applied for the largest cities in Vietnam, such as Hanoi, Danang and Ho Chi Minh city. By this way, the stability and effectiveness of the proposed system can be avaluated easily.

\section{References}

[1] N. Park, N. Kang, "Mutual Authentication Scheme in Secure Internet of Things Technology for Comfortable Lifestyle,” Sensors, vol. 16, 2016. Article (CrossRef Link)

[2] C. Sekhar, K. Kranthi and M. K. Chakravarthi, "Traffic signal breach vehicle stop system using IOT," in Proc. of 2017 International Conference on Nextgen Electronic Technologies: Silicon to Software (ICNETS2), Chennai, pp. 296-300, 2017. Article (CrossRef Link)

[3] T. T. Thakur, A. Naik, S. Vatari and M. Gogate, "Real time traffic management using Internet of Things," in Proc. of 2016 International Conference on Communication and Signal Processing (ICCSP), Melmaruvathur, pp. 1950-1953, 2016. Article (CrossRef Link)

[4] H. F. Chong and D. W. K. Ng, "Development of IoT device for traffic management system," in Proc. of 2016 IEEE Student Conference on Research and Development (SCOReD), Kuala Lumpur, pp. 1-6, 2016. Article (CrossRef Link) 
[5] R. B. Allsop, "SIGSET: A computer program for calculating traffc capacity of signal-controlled road junctions, " Traffic Engeering Control, vol. 12,pp. 58-60, 1971.

[6] M. Papageorgiou, C. Diakaki, V. Dinopoulou, A. Kotsialos, and Y. Wang, "Review of Road Traffic Control Strategies," Proceedings of the IEEE, vol. 91,no.12,pp. 2043-2067, 2003. Article (CrossRef Link)

[7] M. D. Cano, R. Sanchez-Iborra, B. Freire-Viteri, A. J. Garcia-Sanchez, F. Garcia-Sanchez and J. Garcia-Haro, "A self-adaptive approach for traffic lights control in an urban network," in Proc. of 19th International Conference on Transparent Optical Networks (ICTON), Girona, pp. 1-4, 2017. Article (CrossRef Link)

[8] X. Xie, S.F. Smith, L. Lu, and GJ. Barlow, "Schedule-driven intersection control," Transportation Research Part C, vol. 24, pp.168-189,2012 Article (CrossRef Link)

[9] J. D. C. Little, “The sychronization of traffic signals by mixed integerlinear-programming,” Oper. Res.,vol. 14,pp. 568-594,1996. Article (CrossRef Link)

[10] N. H. Gartner,S. F. Assmann,F. Lasaga,and D. 1. Hom, "A multiband approach to arterial traffic signal optimization," Transp. Res. B,vol. 25, pp. 55-74,1991. Article (CrossRef Link)

[11] C. Diakaki, M. Papageorgiou, \& K. Aboudolas, "A multivariable regulator approach to traffic-responsive network-wide signal control," Control Engineering Practice, vol.10, no 2, pp. 183-195, 2002. Article (CrossRef Link)

[12] K. Aboudolas, M. Papageorgiou, \& E. Kosmatopoulos, "Store andforward based methods for the signal control problem in large-scale congested urban road networks," Transportation Research Part C, vol.17, no 2, pp. 163-174, 2009. Article (CrossRef Link)

[13] L. T. Lin, 1. W. Tung, A. M. ASCE and H. C. Ku, "Synchronized signal control model for maximizing progression along arterial," Joural of Tranportation Engineering, vol. 136, No. 8, pp.60-68, 2010. Article (CrossRef Link)

[14] A. K. Mittal and D. Bhandari, "A novel approach to implement green wave system and detection of stolen vehicles," in Proc. of 2013 3rd IEEE International Advance Computing Conference (IACC), Ghaziabad, pp. 1055-1059, 2013. Article (CrossRef Link)

[15] L. W. Chen and C. C. Chang, "Cooperative Traffic Control With Green Wave Coordination for Multiple Intersections Based on the Internet of Vehicles," IEEE Transactions on Systems, Man, and Cybernetics: Systems, vol. 47, no. 7, pp. 1321-1335, July 2017. Article (CrossRef Link)

[16] How Far and How Fast Can You Go with RS-485? Online: https://www.maximintegrated.com/en/app-notes/index.mvp/id/3884

[17] S7 1200 system manual. Online:https://cache.industry.siemens.com/dl/files/465/36932465/att_106119/v1/s71200_system _manual_en-US_en-US.pdf

[18] Modbus Protocol Reference Guide. Online: http://modbus.org/docs/PI_MBUS_300.pdf

[19] Aoxiang Wu, Liqun Qi, Xiaoguang Yang, "Mechanism Analysis and Optimization of Signalized Intersection Coordinated Control under Oversaturated Status,” Procedia - Social and Behavioral Sciences, Volume 96, Pages 1433-1442, 2013. Article (CrossRef Link)

[20] QU Da-yi, WAN Meng-fei, WANG Zi-lin, XU Xiang-hua, WANG Jin-zhan,”Green Wave Coordinate Control Method for Arterial Traffic Based on Traffic Wave Theory,” Journal of Highway and Transponation Research and Development, volume 11, 2017. Article (CrossRef Link)

[21] Bao-Lin Ye, Weimin Wu, and Weijie Mao, "A Method for Signal Coordination in Large-Scale Urban Road Networks,” Mathematical Problems in Engineering, Volume 2015, Article ID 720523, 15 pages, 2015. Article (CrossRef Link)

[22] N.H. Gartner, C. Stamatiadis, "Arterial-based control of traffic flow in urban grid networks," Mathematical and Computer Modelling, Volume 35, Issues 5-6, Pages 657-671, 2002. Article (CrossRef Link)

[23] Kai Lu, Xiaosi Zeng, Lin Li, Jianmin Xu, “Two-Way Bandwidth Maximization Model with Proration Impact Factor for Unbalanced Bandwidth Demands," Journal of Transportation Engineering, Volume 138, Issue 5, May 2012. Article (CrossRef Link) 
[24] M. Papageorgiou, C. Diakaki, V. Dinopoulou, A. Kotsialos, Yibing Wang, "Review of road traffic control strategies,” Proc. of the IEEE , Volume 91, Issue 12, Dec 2003. Article (CrossRef Link)

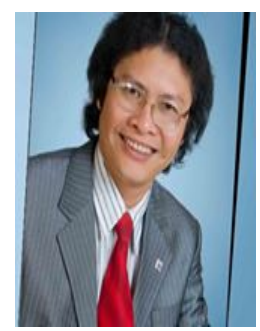

Cao Tho Phan received B. Eng degree in Civil Engineering, M.Sc. degree from the National University of Civil Engineering (NUCE) in 1986 and 1998. He was awarded a Ph.D. degree in Highway and Traffic Engineeng of NUCE (Vietnam), in 2005. Since 1986 to 2014, he has been a Lecturer with The University of Danang - University of Science and Technology (UD-UST), and now he is Rector of The University of Danang University of Technology and Education (UD-UTE), Vietnam. His research interests include Traffic organization and control, Intelligent Traffic System (ITS), Green devolopment and sustainability.

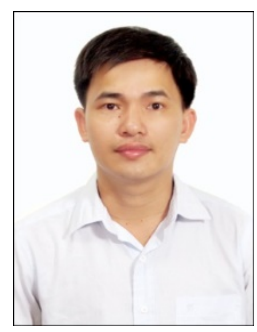

Duy Duong Pham received the master's degree in electrical engineering from the Department of Electrical Engineering, University of Ulsan, Ulsan, South Korea, in 2016. He is currently pursuing the Ph.D. degree with The University of Danang, Danang, Vietnam.

Since 2011, he has been a Lecturer with the The University of Danang - University of Technology and Education, Vietnam. His current research interests include mobile robot control, motion tracking, personal navigation and automation.

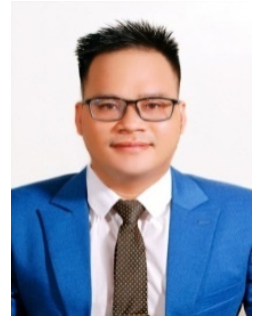

Hoang Vu Tran received B. Eng. degree in Electronics and Telecommunications from Da Nang University of Technology and M.Sc. degree from the University of Danang (Vietnam) in 2004 and 2007, respectively. He was awarded a Ph.D. degree in Electrical Engineering of Hanoi University of Science and Technology (Vietnam), in 2015. Since 2004, he has been a Lecturer with the The University of Danang - University of Technology and Education, Vietnam. His research interests include Reducing power consumption of Data Center Networks, reconfigurable embedded systems, Intelligent Traffic System (ITS) and Internet of Things (IoT).

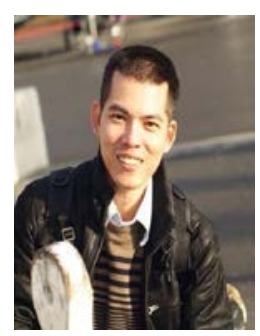

Trung Viet Tran is currently a Lecturer in Faculty of Road and Bridges of The University of Danang- University of Science and Technology (UD-UST), Vietnam. He received his B.Eng from Faculty of Road and Bridges of UD-UST (2007), MEng (2010) and $\mathrm{PhD}$ (2014) from the Ecole Centrale de Nantes, France where his research on reliability-based design of underground structures has influenced spatial variability. Dr. TRAN's main research focus is on risk and reliability in geotechnical and pavement engineering.

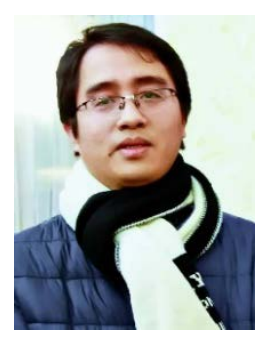

Phat Nguyen Huu received his B.E. (2003), M.S. (2005) degrees in Electronics and Telecommunications at Hanoi University of Sceience and Technology (HUST), Vietnam, and Ph.D. degree (2012) in Computer Science at Shibaura Institute of Technology, Japan. Currently, he lecturer at School of Electronics and Telecommunications, HUST Vietnam. His research interests include digital image and video processing, wireless networks, ad hoc and sensor network, and intelligent traffic system (ITS) and internet of things (IoT). He received the best conference paper award in SoftCOM (2011), best student grant award in APNOMS (2011), hisayoshi yanai honorary award by Shibaura Institute 

traffic lights system for urban areas in Vietnam 